\title{
Transcriptomic profiling of differentially expressed genes and related pathways in different brain regions of Parkinson's disease
}

Qinyu Ge ( $\sim$ geqinyu@seu.edu.cn )

State Key Laboratory of Bioelectronics

Erteng Jia

Southeast University

Min Pan

Southeast University

Zhiyu Liu

Southeast University

Ying Zhou

Southeast University

Xiangwei Zhao

Southeast University

Jian Dong

Southeast University

Yunfei Bai

Southeast University

\section{Research article}

Keywords: Parkinson's disease, Brain, Transcriptomic, Gene expression, Protein-protein interaction networks

Posted Date: December 16th, 2019

DOI: https://doi.org/10.21203/rs.2.18738/v1

License: (1) This work is licensed under a Creative Commons Attribution 4.0 International License. Read Full License 


\section{Abstract}

\section{Background}

Parkinson's disease (PD) is the second most common neurodegenerative disease and many studies have researched its complex pathophysiological processes. However, it is unclear how PD affects the structure of transcripts in different brain regions and how changes in the transcriptomes in different brain regions affect the pathogenesis of PD.

\section{Results}

We generated a PD mouse model by injecting with MPTP solution. RNA sequencing was performed in the cerebral cortex, hippocampus, striatum, and cerebellum regions of the PD mouse. Compared with the control group, these four brain regions showed significant transcriptomic alterations, with the most differentially expressed genes (DEGs) found in the striatum region. The main DEGs were Lrrk2, Mtor, Gxylt1, C920006011Rik, Vdac1, Ano3, Drd4, and Ncan. DEGs were enriched using gene ontology (GO) and Kyoto Encyclopedia of Genes and Genomes enrichment analysis methods, which identified significant GO and molecular pathways. In addition, we used network biology methods to analyze protein-protein relationships, which can accelerate the identification of new PD drugs. The results showed that LRRK2, DRD2, IGF-1, GNAI1, GNAI3, PRKACA, PPP2R5C, and PIK3R1 played a major role in protein regulation.

\section{Conclusions}

Our analysis showed that these DEGs and proteins play an important role in the occurrence and development of PD. Our study also highlighted the potential use of this transcriptomic data for therapeutic strategies and treatment of PD.

\section{Background}

Parkinson's disease (PD) is one of the most common neurological deformations in middle-aged and old people and is the second most common progressive neurodegenerative disorder. The neuropathological hallmarks of PD are related to the loss of dopamine (DA) neurons in the substantia nigra and striatum (ST) [1]. The possible pathogenesis is an increase of oxidative stress in the substantia nigra-ST system, which leads to free radicals cause excessive damage to DA neurons [2]. The clinical symptoms of PD include non-motor and motor disorders. Motor symptoms are generally manifested as hypokinesia, bradykinesia, stiffness, and quiescent tremor [3]. Traditionally, the cerebellum (CB) is associated with motor control. The hippocampus (HP) plays an important role in the interaction between DA transmission and hippocampal synaptic remodeling and, once this interaction is unbalanced, other neuropsychiatric symptoms may occur including dementia [4]. However, non-motor impairment is also manifested by cognitive impairment because neurodegenerative diseases can affect the noradrenergic and cholinergic neurotransmitter systems $[5,6]$. A study by Schapira et al. showed that DA drugs did not alter non-motor disorders. [7]. This view confirms that the pathogenesis of PD is not only related to DA, but also to other 
neurotransmitters. Therefore, the pathogenesis of PD must be studied through differential gene expression and function in different brain regions.

With the advancement of high-throughput sequencing technology and a decrease in cost, RNA-Seq has become a powerful tool for the study of this neurodegenerative disorder and the discovery of biomarkers. Thus, we can research the transcriptomic and biological pathways that are disrupted in PD brains. Recently, there have been many large-scale transcriptomic data related to neurodegenerative diseases found in public databases [8-10]. A study by Alieva et al. (2017) showed significant differences in the expression levels of genes between the ST and substantia nigra in PD [11]. Moni et al. (2019) compared the DEGs in the brain and blood of PD to determine their relationship [12]. Booth et al. showed that leucine-rich repeat kinase 2 (LRRK2) G2019S mutation in the brain can reduce the neuroprotective capacity, thus causing the development of PD pathology [13]. Although PD has been extensively researched, no studies have shown that the disease occurs/develops complete pathways and key differentially expressed genes (DEGs). Therefore, under the current aging worldwide population, it is urgent to study the pathogenesis and control mechanism of PD.

In the present study, we used RNA-Seq to analyze the transcriptome of the cerebral cortex (CC), HP, ST, and $\mathrm{CB}$ on the PD. By analyzing these data, we identified the differential gene expression in each brain region, as well as the main functions and pathways for these differential gene enrichments. These data can provide biologically relevant insights into the occurrence/development of PD, and provide strategies for deciphering the potential diagnosis or prognosis of disease pathogenesis [14].

\section{Methods}

\section{Animals and establishment of PD mouse model}

C57Bl/6J mice (8 weeks old) were included in the present study. All mice were purchased from Shanghai Southern Model Biotechnology Co., Ltd. Mice were maintained at $22^{\circ} \mathrm{C}$ and allowed free access to food and water in a $12 \mathrm{~h}$ dark/light cycle. Eight male mice were randomly divided into a control group and a model group, with four mice in each group. After 3 days of acclimation, the mice in the model group were tested on pole test and rotarod test to collect baseline data. Then, the mice in the model group were intraperitoneally injected with MPTP (Sigma, St. Louis, MO, USA) solution every day at a dose of 20 $\mathrm{mg} / \mathrm{kg}$ for 10 days. The control group mice were injected with normal saline. After 10 days, all the mice in the model group were tested for pole test and rotarod test, and the mice with obvious impairment in their sports ability were screened for subsequent experiments. The study was reviewed and approved by the Ethics Committee of Zhongda Hospital Southeast University.

\section{Sample collection in different brain regions}

The animals were anesthetized and killed immediately after the PD mouse model was established. The mice were anesthetized with tribromoethanol ( $500 \mathrm{mg} / \mathrm{kg}$ ) (Sigma, Saint Louis, USA), and then the mice were killed by cervical dislocation. The control group and PD group were 3 mice respectively. Brain 
samples were dissected to isolate the CC, HP, ST, and CB regions. The mice brain was washed with $0.9 \%$ pre-cooled saline. The long axis of the brain was placed perpendicular to the operator and the CC region was dissected along the middle of the herringbone. The crescent-shaped HP region was clearly seen under the $\mathrm{CC}$ region. The left and right brain were gently opened with tweezers. The ST region was inside and close to the side of the $\mathrm{CC}$ region, with filamentous stripes, similar to serrations. The $\mathrm{CB}$ region was located at the bottom and middle of the brain. The separated mice brain was removed and placed in a 2 $\mathrm{mL}$ enzyme-free EP tube. The brain was immediately frozen in liquid nitrogen and stored at $-80^{\circ} \mathrm{C}$ until subsequent analysis.

\section{Total RNA isolation and cDNA library construction}

Total RNA was isolated from the CC, HP, ST, and CB regions using Trizol reagent (Invitrogen, USA). An Agilent 2100 Bioanalyzer (Agilent Technologies, USA) determined the quality and quantity of RNA. Genomic DNA was removed with RNase-free DNaes I (Qiagen, German). Then, the RNA was purified with $1.8 \times$ magnetic beads (VAHTATM RNA Clean Beads, N412). NEB offered the NEBNext Poly (A) mRNA Magnetic Isolation Module (NEB \#E7490) and the NEBNext rRNA Depletion Kit (NEB \#E6310) for the enrichment of non-ribosomal RNA. The cDNA library was constructed using a NEBNext@ UltraTM II Directional RNA Library Prep Kit for Illumina ${ }^{\circledR}$. The first step was to use reverse transcriptase and random primers to convert the interrupted RNA fragments into the first cDNA strand and the second CDNA strand was synthesized with DNA polymerase I and RNase $\mathrm{H}$. The second step was the end repair process and adapter ligation step, and the adapter was then purified. The third step was the polymerase chain reaction (PCR) enrichment of adaptor-ligated DNA. PCR products were purified by NEBNext Sample Purification Beads. The cDNA library concentration was measured using a Qubit ${ }^{\circledR} 2.0$ fluorometer and each sample was mixed to the same quality. Finally, an Illumina HiSeq 2500 (Illumina, USA) sequencer was used for sequencing.

\section{Analysis of raw data}

FasQC software was used to analyze the quality control of the sequencing data and to obtain the sequencing information of all samples. Then, all sample adapters were removed by Trimmomatic V36 software and clean sequence fragments were obtained. The clean reads of each sample were compared with the reference transcriptome of mice for sequence comparison. The quality of sequencing was assessed by analyzing the ratio of clean reads to reference genomes for each sample.

\section{Differentially expressed genes (DEGs) analysis}

The read counts of the model group were compared with those of the control group. The gene expression between the model group and the control group was compared and analyzed using the DESeq2 R package (1.16.1). More than twice the differential expression and $P<0.05$ were considered as DEGs.

\section{Differentially expression genes enrichment analysis}


Gene ontology (GO) enrichment analysis of DEGs was performed from the Metascape website (http://metascape.org/gp/index.html\#/main/step1). Kyoto Encyclopedia of Genes and Genomes (KEGG) enrichment analysis of DEGs was performed using the David 6.8 website (https://david.ncifcrf.gov/home.jsp). The GO term and KEGG pathways of different brain regions were explored with $P<0.05$ as the significant enrichment. The functions of DEGs and major pathways involved were determined by analyzing the significant enrichment of GO term and KEGG pathways.

\section{Protein-protein interaction (PPI) networks}

In a PPI network, each protein found in an organism's proteome represents a graph node. If there is any interaction between two nodes, then these two nodes are connected. We used STRING online software (http://string-db.org/) to build a PPI network.

\section{Statistical analysis}

The experimental data were analyzed by SPSS 22.0 (SPSS Inc., Chicago, IL, USA) software. One-way ANOVA was used to compare the difference between PD group and control group. Statistical significance was defined as $P<0.05$.

\section{Results}

\section{MPTP-induced PD motor deficits}

We evaluated the exercise capacity of MPTP-induced mice by pole test and rotarod test. Fig. 1A, B showed the time required for the movement of the mice in the pole test and rotarod test. In the pole test, MPTP-induced mice were used longer time than the control group (Fig. 1a). Compared with the control group, the MPTP-induced mice had poor coordination and the rotarod test time was significantly shortened (Fig. 1b). This indicates that MPTP-induced PD mice were successfully constructed.

\section{Summary of RNA-Seq data sets}

To understand the transcriptome information of different brain regions in PD mice, 24 libraries were constructed in the CC, HP, ST, and CB regions. Subsequently, the libraries were sequenced on the Illumina HiSeq 2500 sequencing platform. The raw reads of each sample ranged from 27.4 to 63.2 million (Additional file 1: Table S1). After quality control and filtering, clean reads were obtained. We compared the clean reads to the reference genome with Hisat2. The reads for each gene were calculated using HTSeq-count. Principal component analysis (PCA) was performed based on the DESeq2 method to analyze whether the same brain region samples were together. The results showed that the four brain regions of the control group were divided into different clusters (Fig. 2a). Compared with the CC and HP regions, the transcriptional characteristics of the $\mathrm{CB}$ region were significantly different. In addition, the transcriptome characteristics of the ST were significantly different from those of the CC and HP regions. However, the transcriptome characteristics of the CC and HP regions were not significantly different. The 
four brain regions of the model group were divided into different clusters (Fig. 2b). The difference between the $\mathrm{CB}$ region and the other three brain regions was the most obvious.

\section{Differentially expression gene in different brain regions}

The transcriptome characteristics of the model group and control group mice were analyzed. DEG analysis explained the difference between the transcription in the model group and control group. According to the values of $₫$ fold change $\triangle>2$ and $P<0.05$, DEGs were obtained using the DESeq2 R package.

Cluster analysis was performed using the correlation distance and hierarchical algorithm to show gene expression patterns in different brain regions of the control group and model group (Fig. 3a and b). In the same pathological state, the gene expression in the $\mathrm{CB}$ was significantly different from that in the $\mathrm{CC}, \mathrm{HP}$, and ST regions.

To analyze the effects of gene expression in different brain regions on PD, the DEGs in the model and control groups in the same brain region were compared. As shown in Fig. 3c, 110 DEGs were obtained in the $C C$ region, of which 64 genes were upregulated and 46 genes were downregulated. A total of 179 DEGs were obtained in the HP region, of which 99 genes were upregulated and 80 genes were downregulated. A total of 521 DEGs were obtained in the ST region, of which 485 genes were upregulated and 36 genes were downregulated. There were 96 DEGs in the CB region, of which 67 were upregulated and 29 were downregulated. Fig. 3d shows the overlap of DEGs in the four brain regions. Of the four brain regions, only two genes (Gxylt1 and C920006011 Rik) were co-expressed.

\section{Differentially expressed genes in HP and ST regions of Parkinson mice}

To further understand the transcript information in the HP and ST regions of mice with PD, RNA sequencing analysis was undertaken. Based on the results, unbiased hierarchical clustering analysis was performed for all genes in the HP region. The data of all genes were visualized using a heat map (Fig. 4a). For data quality control, we performed PCA (Fig. 4b). The results showed that the gene expression in the HP region of the model group was different from that in the HP region of the control group. A total of 179 DEGs were found in the HP region in the model group. Additional file 1: Table S2 lists the top 10 upregulated genes and top 10 downregulated genes with the most significant differences in the HP region. In addition, hierarchical clustering analysis of the DEGs showed that there were differences in gene expression in the ST region of the PD model mice (Fig. 4c). Additional file 1: Table S3 lists the top 10 upregulated genes and top 10 downregulated genes with the most significant differences in the ST region.

Compared with the control group, there were 110 (64 upregulated, 46 downregulated) and 96 (67 upregulated, 29 downregulated) DEGs in the $C C$ and $C B$ regions of the model group mice, respectively. Additional file 1: Table S4 and Table S5 listed the top 10 up-regulated genes and the top 10 downregulated genes in $\mathrm{CC}$ and $\mathrm{CB}$ regions, respectively. A heat map of the top 100 genes differentially 
expressed between the control group and model group in the CB region is provided in Additional file 2: Figure S1.

\section{Gene ontology enrichment analysis}

\section{Gene ontology enrichment analysis of the DEGs in HP and ST regions}

The biological functions of DEGs were identified by GO analysis. The GO analysis database can classify DEGs, including "biological processes," "cellular components," and "molecular functions." The GO term corrected by $P<0.05$ was defined as significant enrichment of DEGs. In Fig. 5, the significant enrichment of $\mathrm{GO}$ terms in the three main categories (cellular component, biological process, and molecular function) is shown. In the HP region, the main five subcategories of biological process were "DNA methylation or demethylation," "posttranscriptional regulation of gene expression," "rhythmic process," "protein localization to organelle," and "regulation of DNA metabolic process," and the two subcategories for molecular function were "guanyl-nucleotide exchange factor activity" and "protein serine/threonine kinase activity" (Fig. 5a). In the ST region, the main five subcategories of cell component were "post synapse," "dendritic tree," "presynapse," "perikaryon", and "endoplasmic reticulum subcompartment." The five subcategories for biological processes were "positive regulation of nervous system development," "synapse organization," "regulation of transmembrane transport," "regulation of exocytosis," and "regulation of intracellular transport"; and five subcategories for molecular function, which are "protein domain specific binding," "protein kinase binding," "protein heterodimerization activity," "GABA receptor binding," and "guanyl-nucleotide binding" (Fig. 5d). Fig. 5b, 5c and 5e, 5f show the top 20 clusters of significantly enriched terms in the HP and ST regions, respectively.

\section{Gene ontology enrichment analysis of the DEGs in CC and CB regions}

GO enrichment analysis was performed on DEGs in the $C C$ and $C B$ regions to determine the effects of PD on these two brain regions. The results showed that $10 \mathrm{GO}$ terms were enriched in the $\mathrm{CC}$ region (Fig. 6a), in which the cell components were significantly enriched in two GO terms ("axon" and "filopodium"), the biological process was significantly enriched in six GO terms ("negative regulation of protein kinase activity," "regulation of neuron death," "negative regulation of cell cycle," "ribosome biogenesis," "chromatin organization," and "organophosphate biosynthetic process"), and the molecular function was significantly enriched in two GO terms ("myosin binding" and "lipase activity"). A total of 14 GO terms were enriched in the $\mathrm{CB}$ region (Fig. 6b), in which the cellular components were significantly enriched in two GO terms, the biological process was significantly enriched in nine GO terms, and the molecular function was significantly enriched in three GO terms. In the CC region, the top five significantly enriched GO terms were "axon," "associative learning," "myosin binding," "negative regulation of protein kinase activity," and "regulation of neuron death" (Fig. 6c). In the CB region, the top five significantly enriched GO terms were "negative regulation of transmembrane transport," "translation factor activity, RNA binding," "histone lysine methylation," "response to glucose," and "dioxygenase activity" (Fig. 6d).

\section{KEGG enrichment analysis}


KEGG pathway analysis of DEGs in the HP and ST regions of the model group helped identify the biological pathways related to DEGs. The $P<0.05$ pathway was defined as the DEG enrichment pathway. A total of 179 and 521 DEGs in the HP and ST regions were enriched in 122 and 213 KEGG pathways, respectively. Table 1 shows the pathways and related genes that were significantly enriched in the HP region. In the ST region, the top 10 significantly enriched signaling pathways and related genes are shown in Table 2. There were three signaling pathways ("dopaminergic synapse," "glutamatergic synapse," and "adrenergic signaling in cardiomyocytes"), which was consistent with the study by Fu et al. (2019). However, only a few KEGG pathways were enriched in the CC and CB regions. Only the "focal adhesion" and "regulation of actin cytoskeleton" pathways were significantly enriched in the CC region (Table 3), and only the "adrenergic signaling in cardiomyocytes" and "RNA transport" pathways were significantly enriched in the CB region (Table 3 ).

\section{PPI networks}

There were 427 proteins in the PPI network and the nodes represented proteins (Fig. 7a). The most interacting proteins in the PPI network were LRRK2, DA receptor D2 (DRD2), protein kinase A catalytic subunits (PRKACA), PPP2R5C, insulin-like growth factor 1 (IGF-1), PIK3R1, guanine nucleotide binding protein alpha inhibiting 1 (GNAI1), and guanine nucleotide binding protein alpha inhibiting 3 (GNAl3). A total of 15 proteins that may be associated with PD were extracted from the PPI network and the protein network interactions were reconstructed (Fig. 7b).

\section{Discussion}

Analysis of DEGs in the different brain regions of PD by RNA-Seq can better explain the complexity of gene function and the biological pathways involved. This is the first comprehensive study that examines the gene expression changes in four brain regions. The present study aimed to investigate the changes of gene expression in pathological pathways in different brain regions of PD mice. DEGs screened from different brain regions can be used as biomarkers or therapeutic targets. Previous studies mainly focus on the transcriptome analysis of ST and substantia nigra [16], but less on HP, CC and CB regions.

Emerging data suggest interactions between the dopaminergic systems and the hippocampus in synaptic plasticity, adaptive memory, and motivated behaviour. Therefore, it is important to study the pathological changes in the HP for the study of cognitive dysfunction in PD.

In the present study, the expression of genes in the $\mathrm{CB}$ region was significantly different from that in the HP, ST, and CC regions. Previous studies have shown that a-synuclein was found in the CB of PD and its expression level was significantly decreased [17-20]. Thus, this suggests that regulation of genes and proteins in the CB region may affect Parkinson's disease. Middleton and Strick showed that the CBthalamus-CC pathway affected motor and cognitive functions and was widely connected with the $\mathrm{CC}$ via specific pathways [21]. However, the specific mechanism requires further study. Ichinohe et al. also confirmed that there was a specific pathway between the CB and ST in rats, that is, the cerebellothalamo-motor cortico-striatal pathway and the cerebello-thalamo-striatal pathway affect the 
function of the ST [22]. Therefore, it is necessary to study the differentially expressed genes in CC, HP, ST and $\mathrm{CB}$ brain region and analyze their biological functions.

In the present study, the main DEGs were Lrrk2, Mtor, Gxylt1, C920006011Rik, Vdac1, Ano3, Drd4, and Ncan. Several genes have been linked to PD. However, Gxylt1 and C920006011Rik as co-expressed genes in four brain regions have not been reported to date. In the present study, the DEGs were mainly related to DNA methylation or demethylation, brain development, and presynaptic and postsynaptic functions. Many studies have reported that these functions are related to the occurrence of PD [23-27], which is consistent with our results. Wen et al. showed that the brain and blood methylation levels of PD patients significantly changed compared with the control group [23]. This was mainly due to the change in the methylation level caused by TET1 gene mutation, which led to PD [24]. In the nervous system, DNA methylation plays a key role in regulating the cognitive function of the HP. However, the results of the present study showed that the changes in the DNA methylation status were caused by the changes in gene expression of Ctcf, Meg3, Usp9x, Apobec3, and Alkbh1. In addition, DEGs are also involved in the AMP-activated protein kinase (AMPK) signaling pathway, phosphatidylinositide-3'-OH kinase (PI3K)-Akt signaling pathway, mammalian target of rapamycin (mTOR) signaling pathway, gamma-amino butyric acid positive (GABA-ergic) synapse, glutamatergic synapse, and dopaminergic synapse. AMPK, PI3K-Akt, and mTOR signaling pathways are mutually regulated [28]. AMPK can be activated under oxidative stress, thereby inducing neuronal apoptosis and inhibiting the mTOR pathway, which plays a key role in neurodegenerative diseases [29-32]. The PI3K-Akt signaling pathway plays an important role in the development and function of neurons, and is involved in the pathogenesis of PD, and directly affects the DA content in the substantia nigra [33]. Activated PI3K-Akt may positively regulate mTOR. The mTOR signaling pathway plays a regulatory role in the growth and development of neuronal cells, including the extension of axons and formation of dendrites [34,35]. Recent studies have shown that abnormal activity of Akt or abnormal expression of mTOR was associated with the occurrence and development of PD [3639]. This is consistent with our results, i.e., that the expression of Akt and Mtor genes is significantly downregulated. Therefore, our RNA sequence results are reliable. Proper regulation of AMPK/PI3KAkt/mTOR signaling may be a potential strategy for the prevention and treatment of PD. On the other hand, glutamate and GABA-mediated excitatory synaptic transmission and inhibitory synaptic transmission were the basis for maintaining the excitation/inhibition balance of the nervous system. Previous studies have shown that the excitation/inhibition imbalance is a key factor in neurological impairment [40,41], such as PD [42]. The D4 receptors in the cerebral cortex and hippocampal neurons have been shown to regulate GABA transmission [43]. However, in the present study, Drd4 gene expression was significantly increased in the cerebellum. This suggests that the cerebellum, cerebral cortex and hippocampus may affect PD by co-regulating the expression of genes. Therefore, this result once again shows that the differentially expressed genes in different brain regions may cause Parkinson's disease. In summary, the interaction of glutamatergic synapse and Glutamatergic synapse plays an important role in the pathogenesis of PD. Therefore, glutamate and GABA may help to develop more effective neuroprotective therapies and provide potential treatment strategies for PD patients. 
To determine the molecular network regulators that cause PD, we used STRING software to analyze the correlation between the functions of proteins encoded by DEGs. We used the known PD-associated genes as nodes to reconstruct the PPI network map based on DEGs and related proteins reported in the literature. In the present study, the interactions between 427 DEGs encoded proteins were analyzed. LRRK2, DRD2, IGF-1, GNAI1, GNAI3, PRKACA, PPP2R5C, and PIK3R1 were found to interact the most with other proteins. LRRK2 was expressed in various types of intermediate neurons in the ST and CC, including dopaminergic neurons, GABA-ergic neurons, and cholinergic neurons [44]. Many studies have shown a clear clinical correlation between LRRK2 mutations and PD. LRRK2 played an important role in vesicle transport controlling the release of neurotransmitters or proteins [45] and is involved in the regulation of receptor transport $[46,47]$. Beccano-Kelly et al. have shown that the overexpression of LRRK2 can increase the level of DRD2 protein, which played a major role in dopamine receptors [48]. Therefore, the pathogenesis of PD may be caused by alteration in synaptic vesicle trafficking. According to the literature, BAG5 protein has been confirmed to be associated with HSP70, which had been demonstrated to be present in the Lewy body and acts to prevent protein aggregation and neurodegenerative diseases in cells $[49,50]$. BAG5 protein can interact with a series of proteins to enhance the function of PARKIN to isolate protein aggregation and alleviate PARKIN protective protease function. In combination with these functions, BAG5 is a negative regulator of PARKIN and HSP70, which plays a role in promoting the death of dopaminergic neurons in PD. Previous studies have been demonstrated that the COR domain of LRRK2 interacted with PARKIN in HEK293T cells, possibly mediated by a third party, and BAG5 may be one of them [51].

In addition, we found that the interaction of DRD2 and IGF-1 was mediated by GNAI1 in the present study. As a regulator of adenylate cyclase, GNAl1 plays an important role in the cholinergic synaptic pathway. Cholinergic $M$ receptor agonists can induce DA release, while DA regulates acetylcholine release via DA receptors [52]. Alcantara et al. found that DA receptors were directly located in striatal cholinergic neurons, and activated DRD2 inhibited the release of acetylcholine [53]. Ebert et al. showed that the overexpression of IGF-1 was associated with the proliferation of dopaminergic neurons in the rat PD model, which may be due to the interaction of IGF-1 with DA receptors via cholinergic receptors, thus regulating the transport of DA neurotransmitters [54]. From the transcriptome data of the present study, it is speculated that the Prkaca, Ppp2r5c, Pik3r1, and Adcy 1 genes may be related to the imbalance of DA and acetylcholine; however, the specific functions remain unclear and require further study and verification. A thorough understanding of these node proteins will contribute to the study of the pathogenesis of PD and the development of PD therapeutic drugs.

\section{Conclusions}

Our transcriptome analyses on a PD mice model revealed differences in the gene expression patterns in the CC, HP, ST, and CB. This model also identified the key genes and signaling pathways in the different brain regions of PD. According to the functional enrichment of differentially expressed genes in different brain regions, HP and ST were dominant in the occurrence of $\mathrm{PD}$, and $\mathrm{CB}$ and $\mathrm{CC}$ also played a role in the pathological regulation. The present research enhances our understanding of gene regulation of 
transcriptional levels in different brain regions of PD; therefore, it is necessary to explore the pathogenesis and treatment of PD at the molecular level.

\section{Abbreviations}

CC: cerebral cortex; HP: hippocampus; ST: striatum; CB: cerebellum; GO: Gene Ontology; KEGG: Kyoto Encyclopedia of Genes and Genomes; DEGs: differentially expressed genes; PD: Parkinson's disease; DA: dopamine; LRRK2: leucine-rich repeat kinase 2; PPI: Protein-protein interaction; PCA: Principal component analysis; CN: control; AMPK: AMP-activated protein kinase; PI3K: phosphatidylinositide-3'-OH kinase

\section{Declarations}

\section{Acknowledgements}

We thank Changwei Bi for helping us upload the data to NCBI.

\section{Funding}

This work was supported by the grant from the National Natural Science Foundation of China No.81827901 \& No.61801108. The funding bodies played no role in the design of the study and collection, analysis, and interpretation of data, nor in writing the manuscript.

\section{Ethics approval and consent to participate}

All animal research protocols for this work were reviewed and approved by the Ethics Committee of Zhongda Hospital Southeast University.

\section{Availability of data and materials}

The datasets used and/or analyzed during the current study are available from the corresponding author on reasonable request.

\section{Consent for publication}

Not applicable.

\section{Competing interests}

The authors declare that they have no competing interests.

\section{References}


1. Lesage S, Brice A. Parkinson's disease: from monogenic forms to genetic susceptibility factors. Hum Mol Genet. 2009; 18(R1): R48-59.

2. Simchovitz A, Soreq L, Soreq H. Transcriptome profiling in Parkinson's leukocytes: from early diagnostics to neuroimmune therapeutic prospects. Curr Opin Pharmacol. 2016; 26: 102-9.

3. Lang AE, Lozano AM. Parkinson's Disease. Lancet. 1998; 386(9996): 896-912.

4. Calabresi P, Castrioto A, Di Filippo M, Picconi B. New experimental and clinical links between the hippocampus and the dopaminergic system in Parkinson's disease. Lancet Neurol. 2013; 12(8): 81121.

5. Braak H, Del Tredici K, Rüb U, de Vos RA, Jansen Steur EN, Braak E. Staging of brain pathology related to sporadic Parkinson's disease. Neurobiol Aging. 2003; 24(2): 197-211.

6. Hawkes CH, Del Tredici K, Braak H. Parkinson's disease: a dual-hit hypothesis. Neuropathol Appl Neurobiol. 2007; 33(6): 599-614.

7. Schapira AHV, Chaudhuri KR, Jenner P. Non-motor features of Parkinson disease. Nat Rev Neurosci. 2017; 18(7): 435-50.

8. Barrett T, Troup DB, Wilhite SE, Ledoux P, Rudnev D, Evangelista C, et al. NCBI GEO: archive for highthroughput functional genomic data. Nucleic Acids Res. 2009; 37: D885-90.

9. Kang HJ, Kawasawa Y, Cheng F, Zhu Y, Xu X, Li M, et al. Spatio-temporal transcriptome of the human brain. Nature. 2011; 478(7370): 483-9.

10. Jones AR, Overly CC, Sunkin SM. The Allen Brain Atlas: 5 years and beyond. Nat Rev Neurosci. 2009; 10(11): 821-8.

11. Alieva AK, Filatova EV, Kolacheva AA, Rudenok MM, Slominsky PA, Ugrumov MV, et al. Transcriptome Profile Changes in Mice with MPTP-Induced Early Stages of Parkinson's Disease. Mol Neurobiol. 2017; 54(9): 6775-84.

12. Moni MA, Rana HK, Islam MB, Ahmed MB, Xu H, Hasan MAM, et al. A computational approach to identify blood cell-expressed Parkinson's disease biomarkers that are coordinately expressed in brain tissue. Comput Biol Med. 2019; 113: 103385.

13. Booth HDE, Wessely F, Connor-Robson N, Rinaldi F, Vowles J, Browne C, et al. RNA sequencing reveals MMP2 and TGFB1 downregulation in LRRK2 G2019S Parkinson's iPSC-derived astrocytes. Neurobiol Dis. 2019; 129: 56-66.

14. Infante J, Prieto C, Sierra M, Sánchez-Juan P, González-Aramburu I, Sánchez-Quintana C, et al. Identification of candidate genes for Parkinson's disease through blood transcriptome analysis in LRRK2-G2019S carriers, idiopathic cases, and controls. Neurobiol Aging. 2015; 36(2): 1105-9.

15. Fu X, Luo L, Yi R, Ding B, Wang C, Zhang W, et al. Transcriptome profiling in Eid1-KO mice brain shows that Eid1 links cell proliferation in the brain. Gene. 2019; 717: 143998.

16. Alieva AK, Zyrin VS, Rudenok MM, Kolacheva AA, Shulskaya MV, Ugryumov MV, et al. WholeTranscriptome Analysis of Mouse Models with MPTP-Induced Early Stages of Parkinson's Disease 
Reveals Stage-Specific Response of Transcriptome and a Possible Role of Myelin-Linked Genes in Neurodegeneration. Mol Neurobiol. 2018; 55(9): 7229-41.

17. Solano SM, Miller DW, Augood SJ, Young AB, Penney JB Jr. Expression of alpha-synuclein, parkin, and ubiquitin carboxy-terminal hydrolase L1 mRNA in human brain: genes associated with familial Parkinson's disease. Ann Neurol. 2000; 47(2): 201-10.

18. Piao YS, Mori F, Hayashi S, Tanji K, Yoshimoto M, Kakita A, et al. Alpha-synuclein pathology affecting Bergmann glia of the cerebellum in patients with alpha-synucleinopathies. Acta Neuropathol. 2003; 105(4): 403-9.

19. Fuchs J, Tichopad A, Golub Y, Munz M, Schweitzer KJ, Wolf B, et al. Genetic variability in the SNCA gene influences alpha-synuclein levels in the blood and brain. FASEB J. 2008; 22(5): 1327-34..

20. Westerlund M, Belin AC, Anvret A, Håkansson A, Nissbrandt H, Lind C, et al. Cerebellar alphasynuclein levels are decreased in Parkinson's disease and do not correlate with SNCA polymorphisms associated with disease in a Swedish material. FASEB J. 2008; 22(10): 3509-14.

21. Middleton FA, Strick PL. Basal ganglia and cerebellar loops: motor and cognitive circuits. Brain Res Brain Res Rev. 2000; 31(2-3): 236-50.

22. Ichinohe N, Mori F, Shoumura K. A di-synaptic projection from the lateral cerebellar nucleus to the laterodorsal part of the striatum via the central lateral nucleus of the thalamus in the rat. Brain Res. 2000; 880(1-2): 191-7.

23. Wen KX, Miliç J, El-Khodor B, Dhana K, Nano J, Pulido T, et al. The Role of DNA Methylation and Histone Modifications in Neurodegenerative Diseases: A Systematic Review. PLoS One. 2016; 11(12): e0167201.

24. Shu L, Qin L, Min S, Pan H, Zhong J, Guo J, et al. Genetic analysis of DNA methylation and hydroxymethylation genes in Parkinson's disease. Neurobiol Aging. 2019; S0197-4580(19): 30075-2.

25. Calabresi P, Galletti F, Saggese E, Ghiglieri V, Picconi B. Neuronal networks and synaptic plasticity in Parkinson's disease: beyond motor deficits. Parkinsonism Relat Disord. 2007; 13: S259-62.

26. Lee S, Liu HP, Lin WY, Guo H, Lu B. LRRK2 kinase regulates synaptic morphology through distinct substrates at the presynaptic and postsynaptic compartments of the Drosophila neuromuscular junction. J Neurosci. 2010; 30(50): 16959-69.

27. Di Maio R, Hoffman EK, Rocha EM, Keeney MT, Sanders LH, De Miranda BR, et al. LRRK2 activation in idiopathic Parkinson's disease. Sci Transl Med. 2018; 10(451). pii: eaar5429.

28. Xu Y, Liu C, Chen S, Ye Y, Guo M, Ren Q, et al. Activation of AMPK and inactivation of Akt result in suppression of mTOR-mediated S6K1 and 4E-BP1 pathways leading to neuronal cell death in in vitro models of Parkinson's disease. Cell Signal. 2014; 26(8): 1680-9.

29. Hardie DG. AMP-activated/SNF1 protein kinases: conserved guardians of cellular energy. Nat Rev Mol Cell Biol. 2007; 8(10): 774-85.

30. Chen L, Xu B, Liu L, Luo Y, Yin J, Zhou H, et al. Hydrogen peroxide inhibits mTOR signaling by activation of AMPKalpha leading to apoptosis of neuronal cells. Lab Invest. 2010; 90(5): 762-73. 
31. Jiang P, Gan M, Ebrahim AS, Castanedes-Casey M, Dickson DW, Yen SH. Adenosine monophosphateactivated protein kinase overactivation leads to accumulation of a-synuclein oligomers and decrease of neurites. Neurobiol Aging. 2013; 34(5): 1504-15.

32. Vingtdeux V, Davies P, Dickson DW, Marambaud P. AMPK is abnormally activated in tangle- and pretangle-bearing neurons in Alzheimer's disease and other tauopathies. Acta Neuropathol. 2011; 121(3): 337-49.

33. Jha SK, Jha NK, Kar R, Ambasta RK, Kumar P. p38 MAPK and PI3K/AKT Signalling Cascades inParkinson's Disease. Int J Mol Cell Med. 2015; 4(2): 67-86.

34. Kumar V, Zhang MX, Swank MW, Kunz J, Wu GY. Regulation of dendritic morphogenesis by Ras-PI3KAkt-mTOR and Ras-MAPK signaling pathways. J Neurosci. 2005; 25(49): 11288-99.

35. Jaworski J, Spangler S, Seeburg DP, Hoogenraad CC, Sheng M. Control of dendritic arborization by the phosphoinositide-3'-kinase-Akt-mammalian target of rapamycin pathway. J Neurosci. 2005; 25(49): 11300-12.

36. Malagelada C, Ryu EJ, Biswas SC, Jackson-Lewis V, Greene LA. RTP801 is elevated in Parkinson brain substantia nigral neurons and mediates death in cellular models of Parkinson's disease by a mechanism involving mammalian target of rapamycin inactivation. J Neurosci. 2006; 26(39): 999610005.

37. Chong ZZ, Shang YC, Wang S, Maiese K. Shedding new light on neurodegenerative diseases through the mammalian target of rapamycin. Prog Neurobiol. 2012; 99(2): 128-48.

38. Timmons S, Coakley MF, Moloney AM, O' Neill C. Akt signal transduction dysfunction in Parkinson's disease. Neurosci Lett. 2009; 467(1): 30-5.

39. Khurana V, Lu Y, Steinhilb ML, Oldham S, Shulman JM, Feany MB. TOR-mediated cell-cycle activation causes neurodegeneration in a Drosophila tauopathy model. Curr Biol. 2006; 16(3): 230-41.

40. Ouyang C, Guo L, Lu Q, Xu X, Wang H. Enhanced activity of GABA receptors inhibits glutamate release induced by focal cerebral ischemia in rat striatum. Neurosci Lett. 2007; 420(2): 174-8.

41. Pozdnyakova N, Yatsenko L, Parkhomenko N, Himmelreich N. Perinatal hypoxia induces a longlasting increase in unstimulated gaba release in rat brain cortex and hippocampus. The protective effect of pyruvate. Neurochem Int. 2011; 58(1): 14-21.

42. Barone P. Neurotransmission in Parkinson's disease: beyond dopamine. Eur J Neurol. 2010; 17(3): 364-76.

43. Tsuchimine S, Yasui-Furukori N, Kaneda A, Saito M, Sugawara N, Kaneko S. Minor genetic variants of the dopamine D4 receptor (DRD4) polymorphism are associated with novelty seeking in healthy Japanese subjects. Prog Neuropsychopharmacol Biol Psychiatry. 2009; 33(7): 1232-5.

44. Higashi S, Moore DJ, Colebrooke RE, Biskup S, Dawson VL, Arai H, et al. Expression and localization of Parkinson's disease-associated leucine-rich repeat kinase 2 in the mouse brain. J Neurochem. 2007; 100(2): 368-81.

45. Rassu M, Del Giudice MG, Sanna S, Taymans JM, Morari M, Brugnoli A, et al. Role of LRRK2 in the regulation of dopamine receptor trafficking. PLoS One. 2017; 12(6): e0179082. 
46. Sanna G, Del Giudice MG, Crosio C, laccarino C. LRRK2 and vesicle trafficking. Biochem Soc Trans. 2012; 40(5): 1117-22.

47. Esposito G, Ana Clara F, Verstreken P. Synaptic vesicle trafficking and Parkinson's disease. Dev Neurobiol. 2012; 72(1): 134-44.

48. Beccano-Kelly DA, Volta M, Munsie LN, Paschall SA, Tatarnikov I, Co K, et al. LRRK2 overexpression alters glutamatergic presynaptic plasticity, striatal dopamine tone, postsynaptic signal transduction, motor activity and memory. Hum Mol Genet. 2015; 24(5): 1336-49.

49. Auluck PK, Chan HY, Trojanowski JQ, Lee VM, Bonini NM. Chaperone suppression of alpha-synuclein toxicity in a Drosophila model for Parkinson's disease. Science. 2002; 295(5556): 865-8.

50. McLean PJ, Kawamata H, Shariff S, Hewett J, Sharma N, Ueda K, et al. TorsinA and heat shock proteins act as molecular chaperones: suppression of alpha-synuclein aggregation. J Neurochem. 2002; 83(4): 846-54.

51. Venderova K, Kabbach G, Abdel-Messih E, Zhang Y, Parks RJ, Imai Y, et al. Leucine-Rich Repeat Kinase 2 interacts with Parkin, DJ-1 and PINK-1 in a Drosophila melanogaster model of Parkinson's disease. Hum Mol Genet. 2009; 18(22): 4390-404.

52. Gray JA, Roth BL. Molecular targets for treating cognitive dysfunction in schizophrenia. Schizophr Bull. 2007; 33(5): 1100-19.

53. Alcantara AA, Chen V, Herring BE, Mendenhall JM, Berlanga ML. Localization of dopamine D2 receptors on cholinergic interneurons of the dorsal striatum and nucleus accumbens of the rat. Brain Res. 2003; 986(1-2): 22-9.

54. Ebert AD, Beres AJ, Barber AE, Svendsen CN. Human neural progenitor cells over-expressing IGF-1 protect dopamine neurons and restore function in a rat model of Parkinson's disease. Exp Neurol. 2008; 209(1): 213-23.

\section{Tables}

Table 1. KEGG enrichment signaling pathways and significant genes in HP region. 
AMPK signaling pathway

Elavl1, Eef2, Mtor, Ppp2ca, Ppp2r3d, Rps6kb2, Akt3

PI3K-Akt signaling pathway Jak1, Mtor, Ppp2ca, Ppp2r3d, Rps6kb2, Akt3, Ywhab

Oocyte meiosis Ppp2ca, Ppp3r1, Smc3, Ywhab

Hepatitis C Jak1, Eif2s1, Ppp2ca, Akt3

Acute myeloid leukemia

Mtor, Rps6kb2, Akt3

mTOR signaling pathway

Fzd1, Akt3, Mtor, Rps6kb2

ErbB signaling pathway

Mtor, Rps6kb2, Akt3

Table 2. KEGG enrichment signaling pathways and significant genes in ST region. 
Signaling pathway Genes

GABA-ergic synapse Abat, Nsf, Adcy1, Adcy2, Gabrb1, Gnai1, Gnai3, Gng2, Prkca, Prkcb, Prkaca, Slc32a1

Morphine addiction Adcy1, Adcy2, Gabrb1, Gnai1, Gnai3, Gng2, Oprm1, Pde7b, Prkca, Prkcb, Prkaca, Slc32a1

Retrograde Adcy1, Adcy2, Gabrb1, Gria1, Grm5, Gnai1, Gnai3, Gng2, Prkca, endocannabinoid Prkcb, Prkaca, Slc32a1

signaling

Gap junction

Adcy1, Adcy2, Drd2, Grm5, Gnai1, Gnai3, Prkca, Prkcb, Prkaca, Tuba1a, Tubb2a

Cholinergic synapse Bcl2, Adcy1, Adcy2, Chrna4, Gnai1, Gnai3, Gng2, Pik3r1, Kcnj4, Prkca, Prkcb, Prkaca

Glutamatergic Adcy1, Adcy2, Gria1, Grin3b, Grik3, Grm5, Gnai1, Gnai3, Gng2, synapse Prkca, Prkcb, Prkaca

Dopaminergic Drd2, Gria1, Gnai1, Gnai3, Gng2, Kif5a, Prkca, Prkcb, Prkaca, synapse Ppp1cb, Ppp2ca, Ppp2r5c

Oocyte meiosis Adcy1, Adcy2, Cdc27, Igf1r, Prkaca, Ppp1cb, Ppp2ca, Ppp2r5c, Smc3, Ywhae, Ywhag

Endocytosis Arfgef2, Arap2, Cbl, Rab11fip4, Capza1, Chmp4b, Chmp5, Cltb, Dnm3, Eea1, Igf1r, Kif5a, Mvb12b, Nedd4l, Prkci, Rbsn, Smap2 Adrenergic Bcl2, Adcy1, Adcy2, Gnai1, Gnai3, Myh7, Prkca, Prkaca, Ppp1cb, signaling in Ppp2ca, Ppp2r5c, Scn1b cardiomyocytes

Table 3. KEGG enrichment signaling pathways and significant genes in CC and CB regions. 


\begin{tabular}{lll}
\hline & Signaling pathway & Genes \\
\hline CC & Focal adhesion & Capn2, Ilk, Myl12b \\
& Regulation of actin cytoskeleton & Myl12b, Arhgef4, Gm5741 \\
CB & Adrenergic signaling in cardiomyocytes & Atp1a2, Ppp2ca, Scn1b \\
& RNA transport & Eef1a2, Eif2b1, Eif2b4 \\
\hline
\end{tabular}

Figures
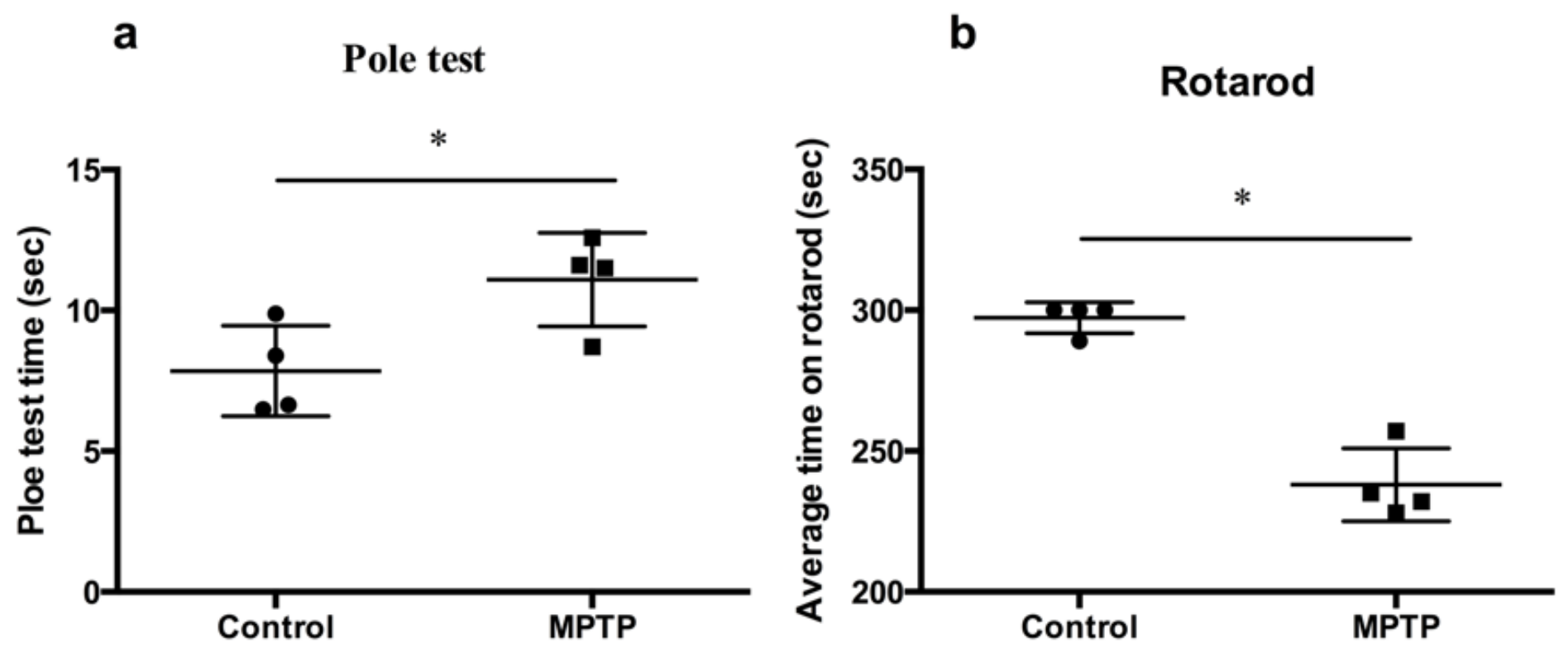

Figure 1

Effect of PD on Pole test and Rotarod test. 
a

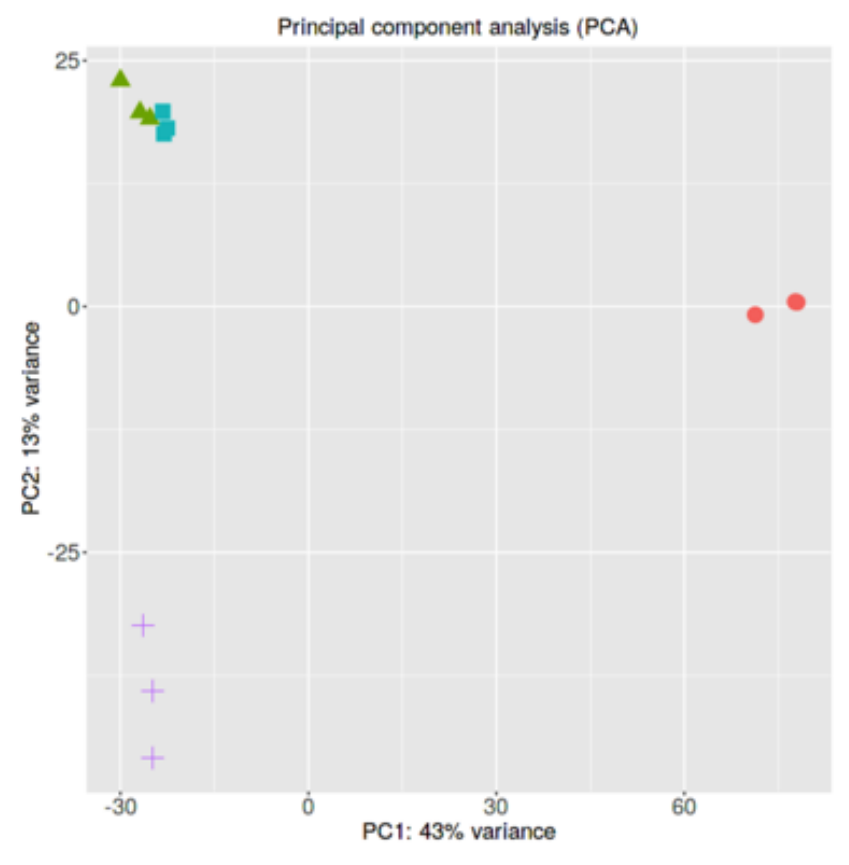

b

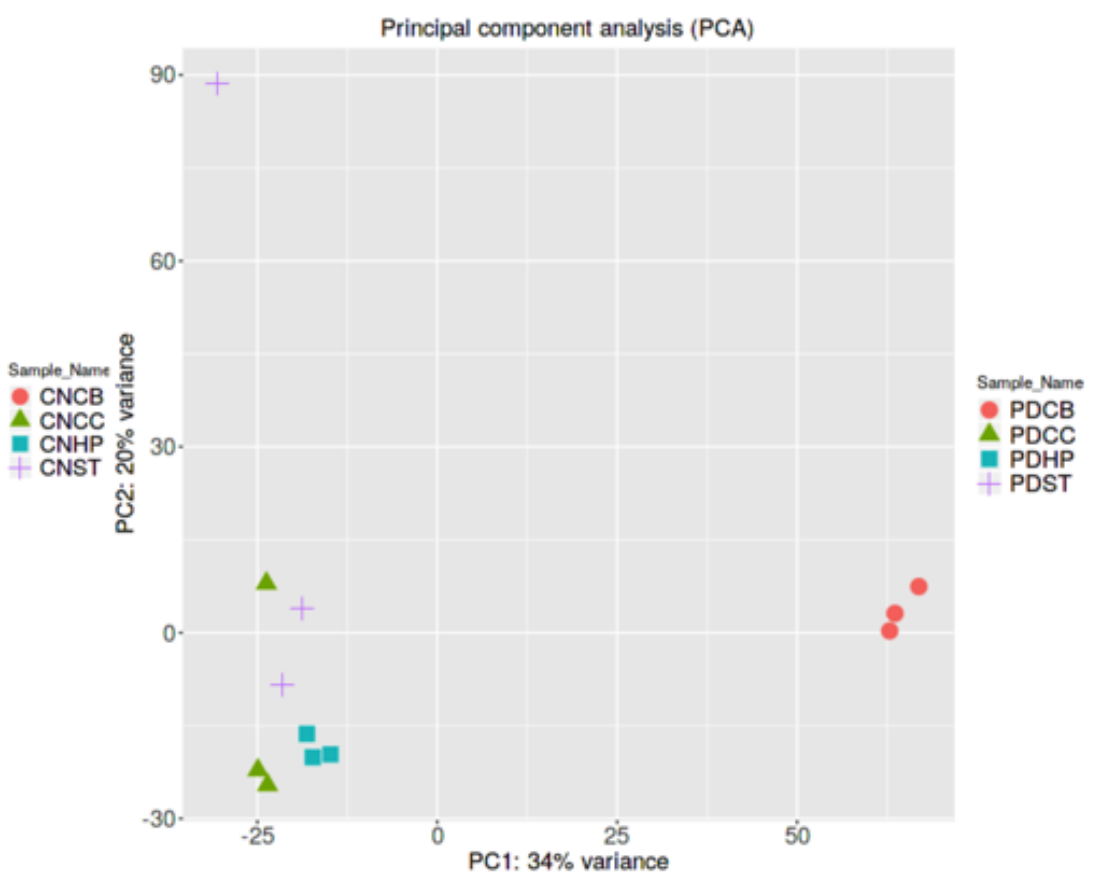

\section{Figure 2}

Different brain regions samples principal component analysis. 
a
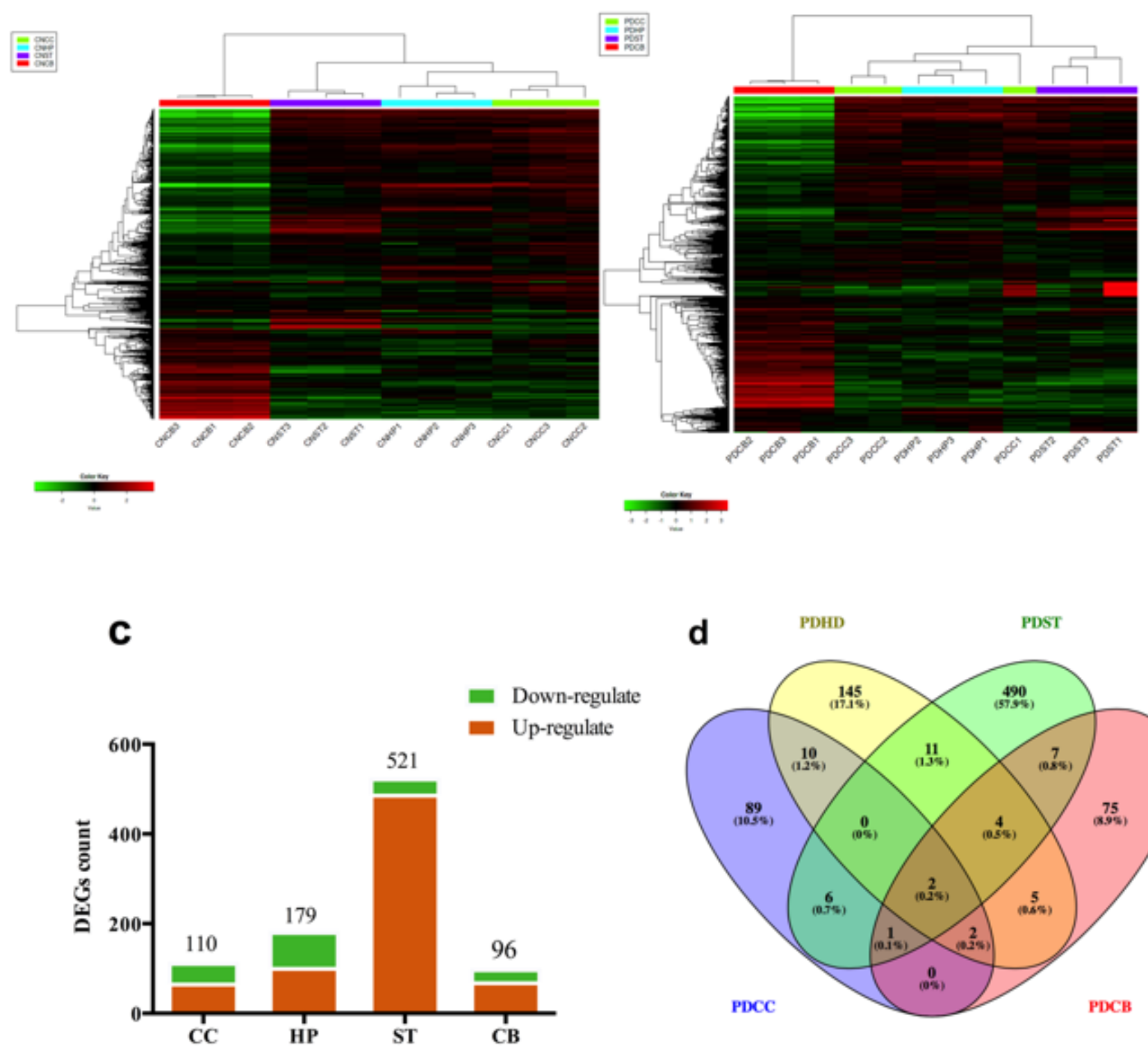

d

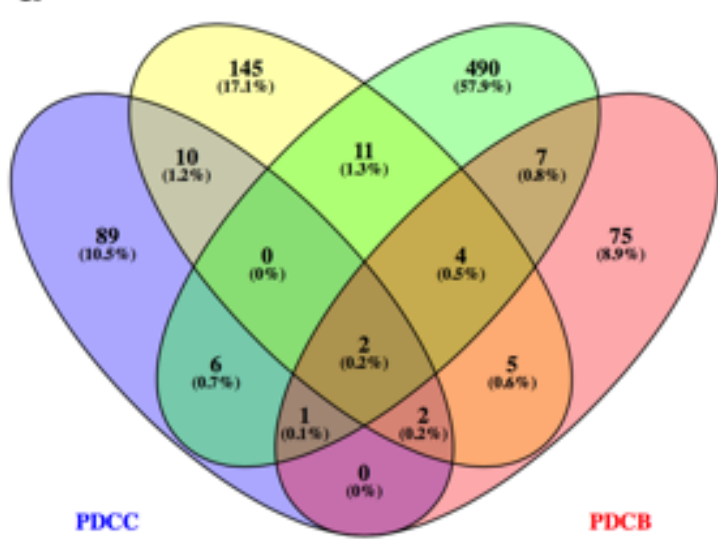

Figure 3

Transcriptomic Profiling of the PD. 
a
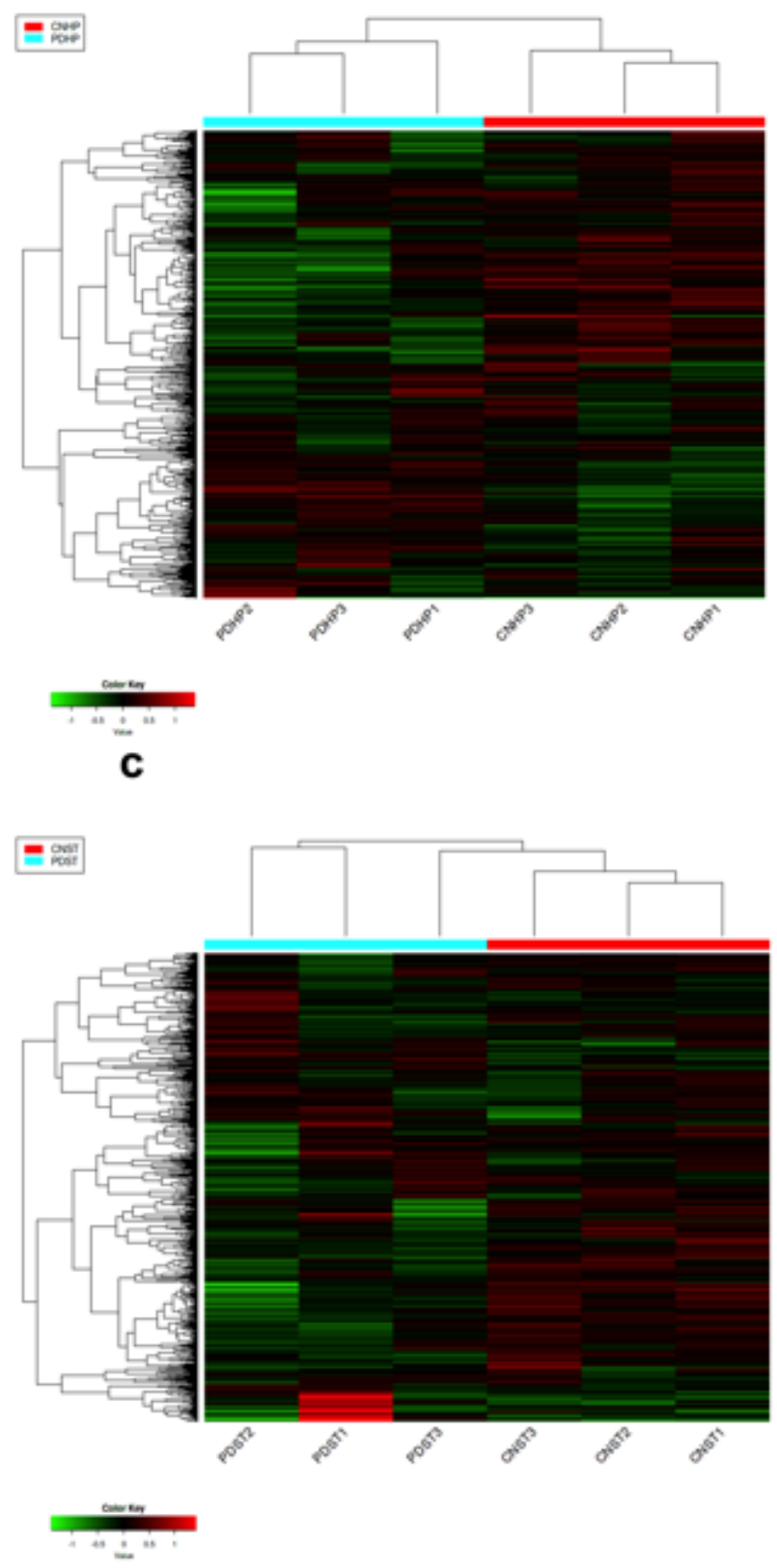

Figure 4

Heat map of gene expression and principal component analysis. 
a

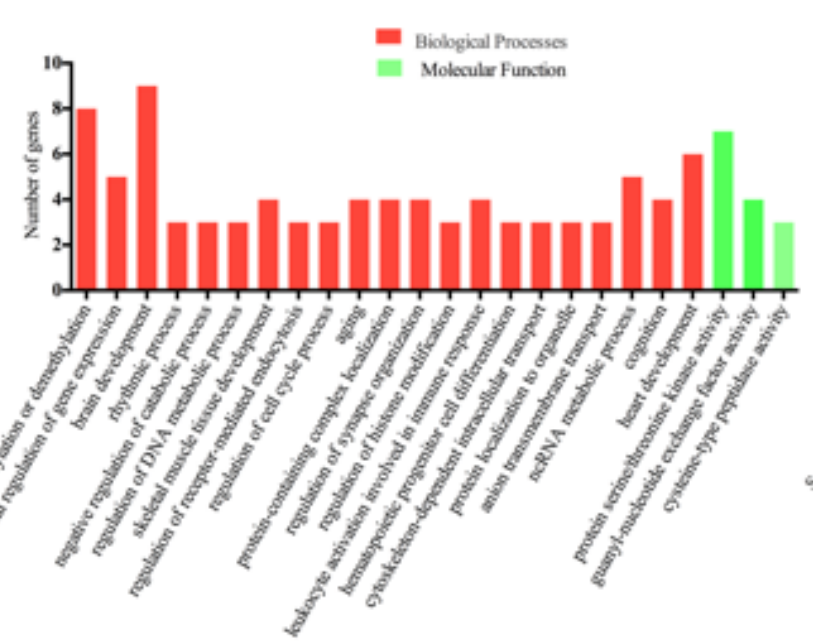

b

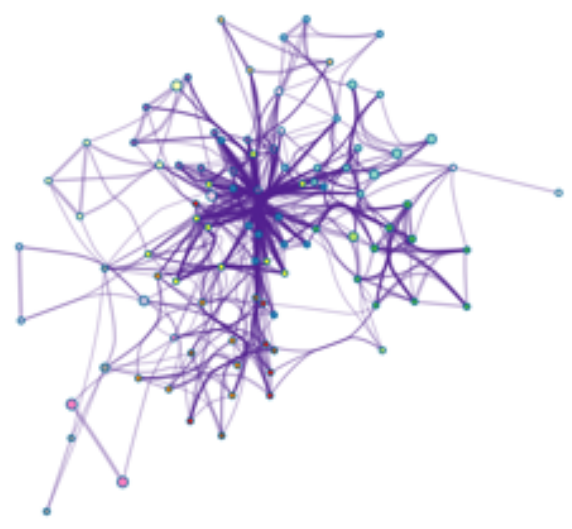

C

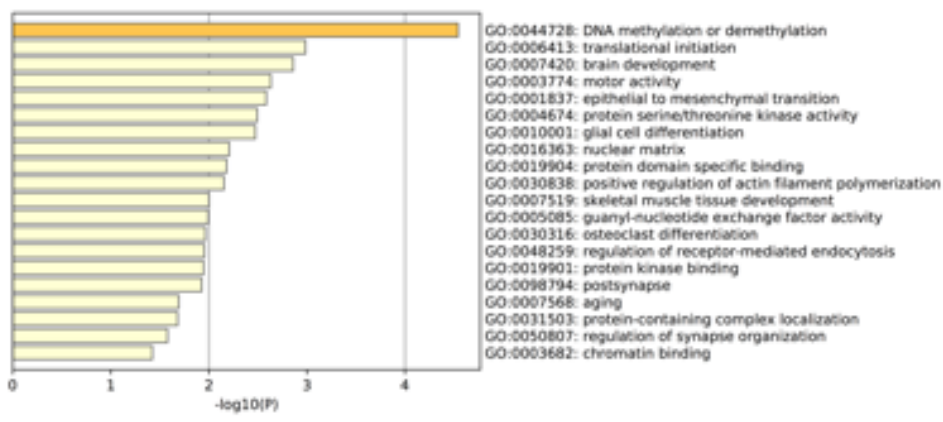

d
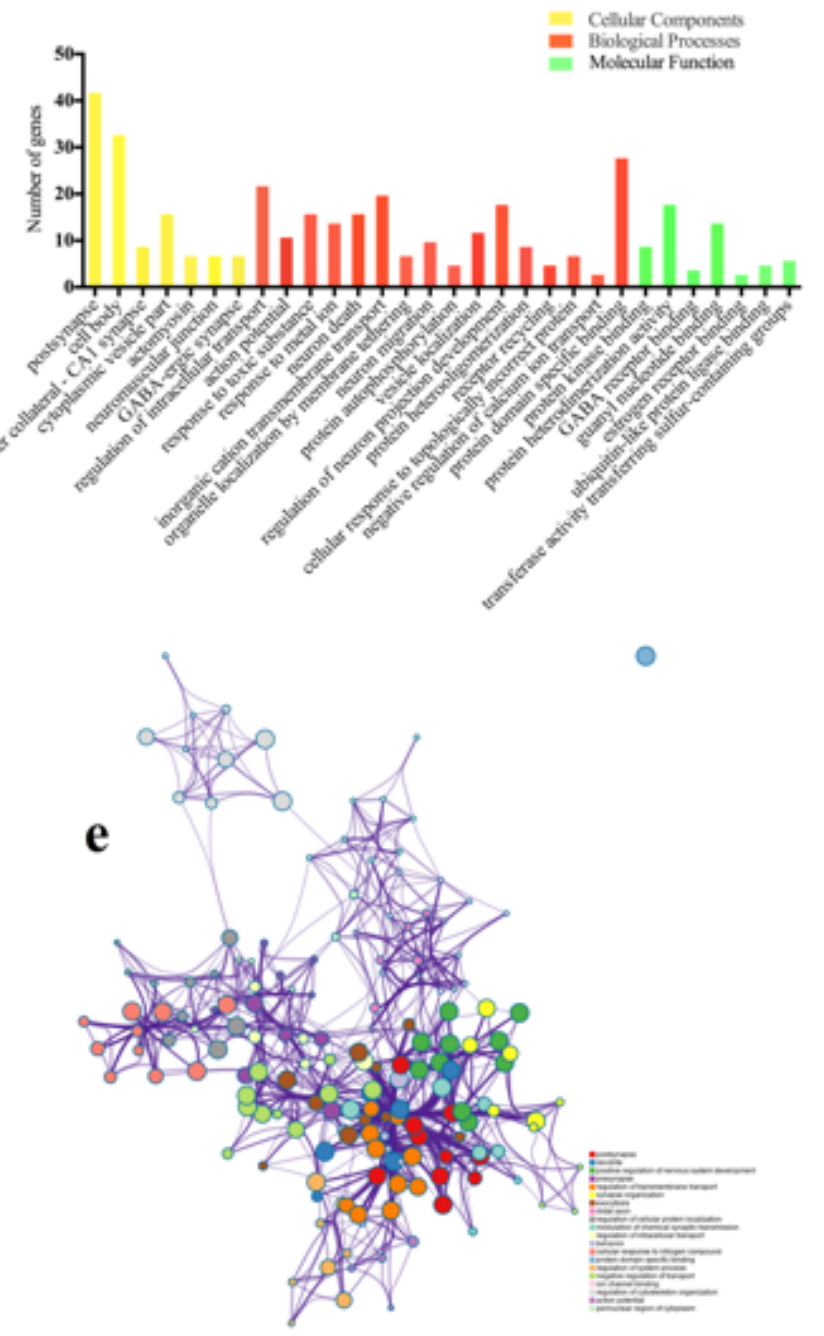

f

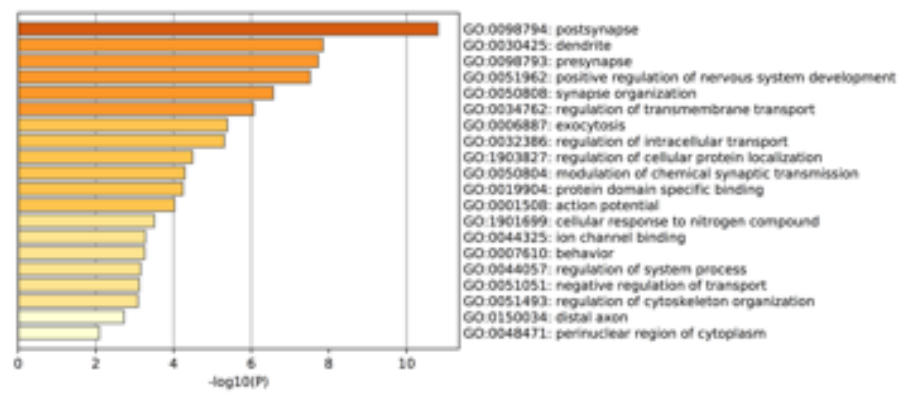

Figure 5

GO enrichment analysis of the DEGs in HP and ST regions. 
a

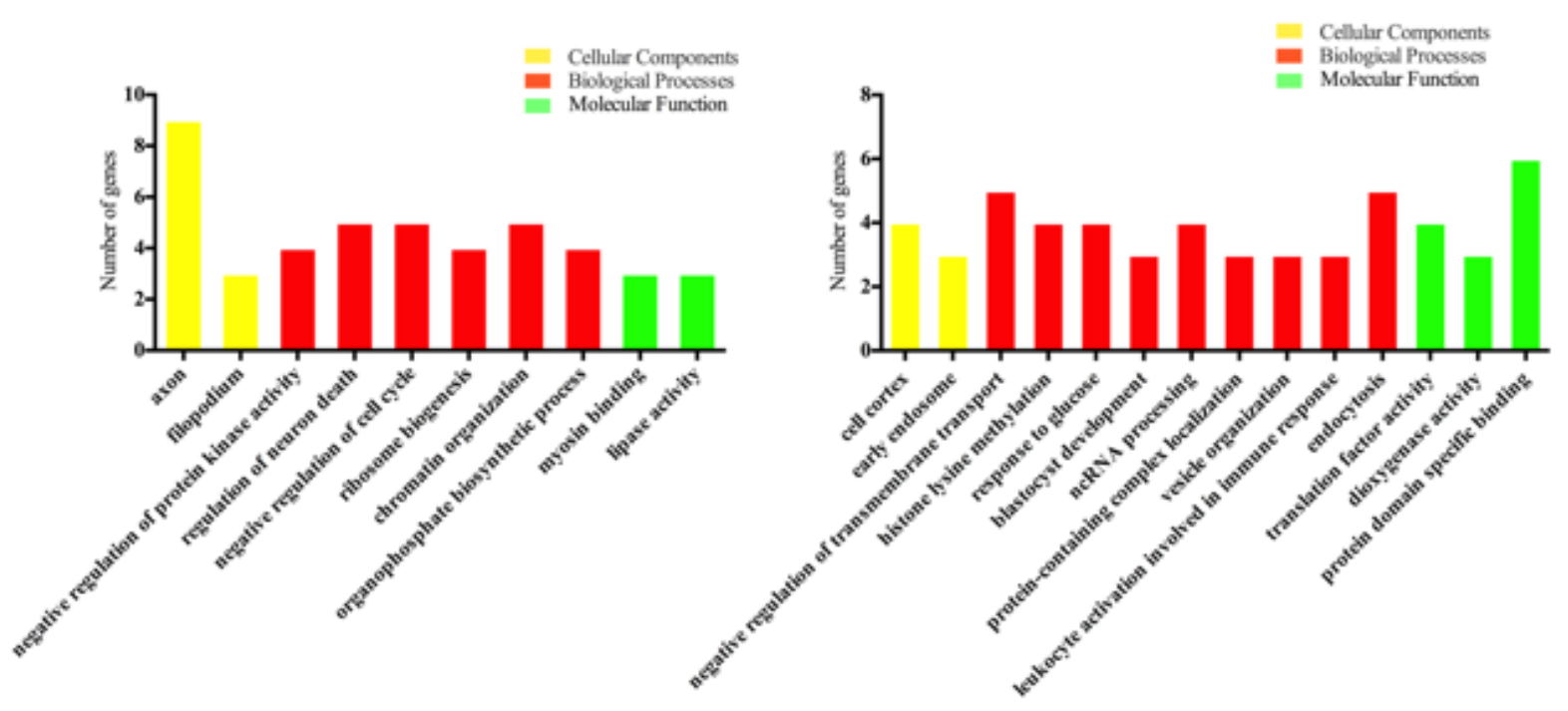

c

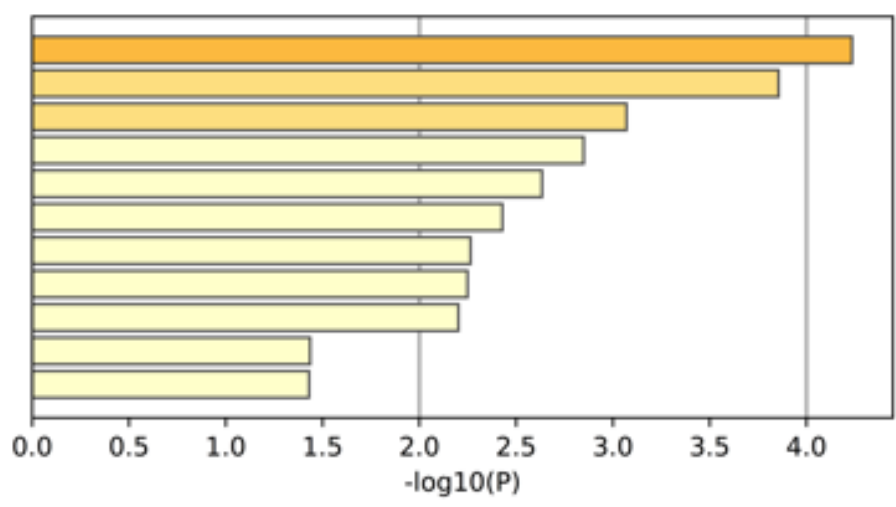

GO:0030424: axon

GO:0008306: associative learning

GO:0017022: myosin binding

GO:0006469: negative regulation of protein kinase activity

GO:1901214: regulation of neuron death

GO:0016298: lipase activity

GO:0045786: negative regulation of cell cycle

GO:0048285: organelle fission

GO:0042254: ribosome biogenesis

GO:0006325: chromatin organization

GO:0090407: organophosphate biosynthetic process

\section{d}

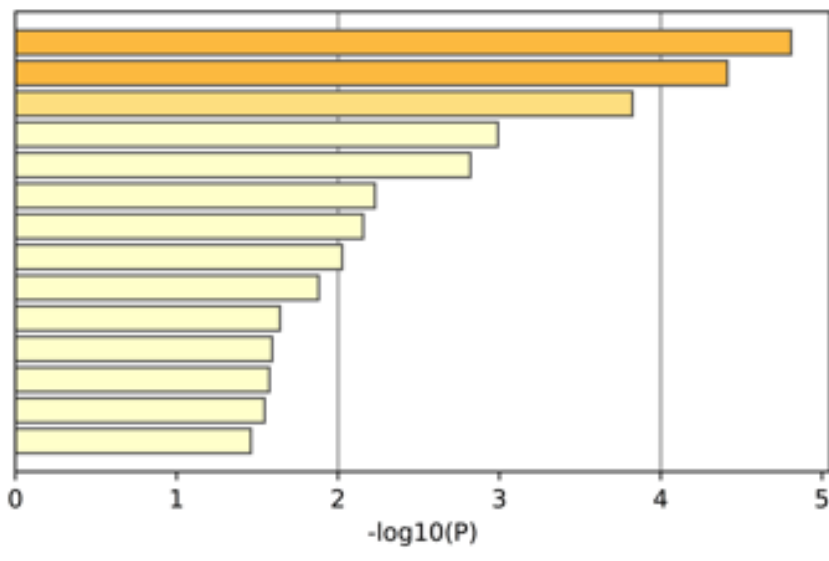

GO:0034763: negative regulation of transmembrane transport GO:0008135: translation factor activity, RNA binding GO:0034968: histone lysine methylation

GO:0009749: response to glucose

GO:0051213: dioxygenase activity

GO:0001824: blastocyst development

GO:0005938: cell cortex

GO:0034470: ncRNA processing

GO:0019904: protein domain specific binding

GO:0031503: protein-containing complex localization

GO:0016050: vesicle organization

GO:0005769: early endosome

GO:0002366: leukocyte activation involved in immune response

GO:0006897: endocytosis

\section{Figure 6}

GO enrichment analysis of the DEGs in CC and CB regions. 
a

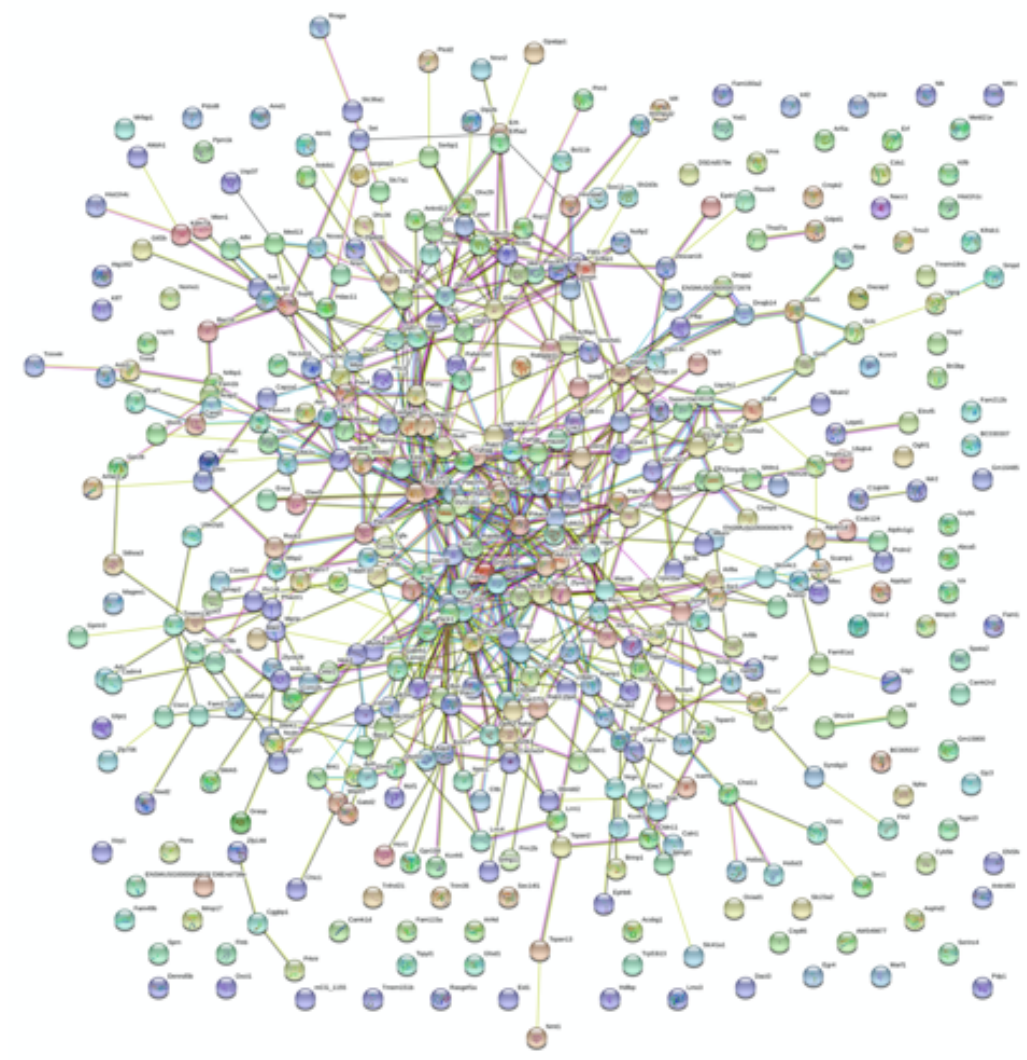

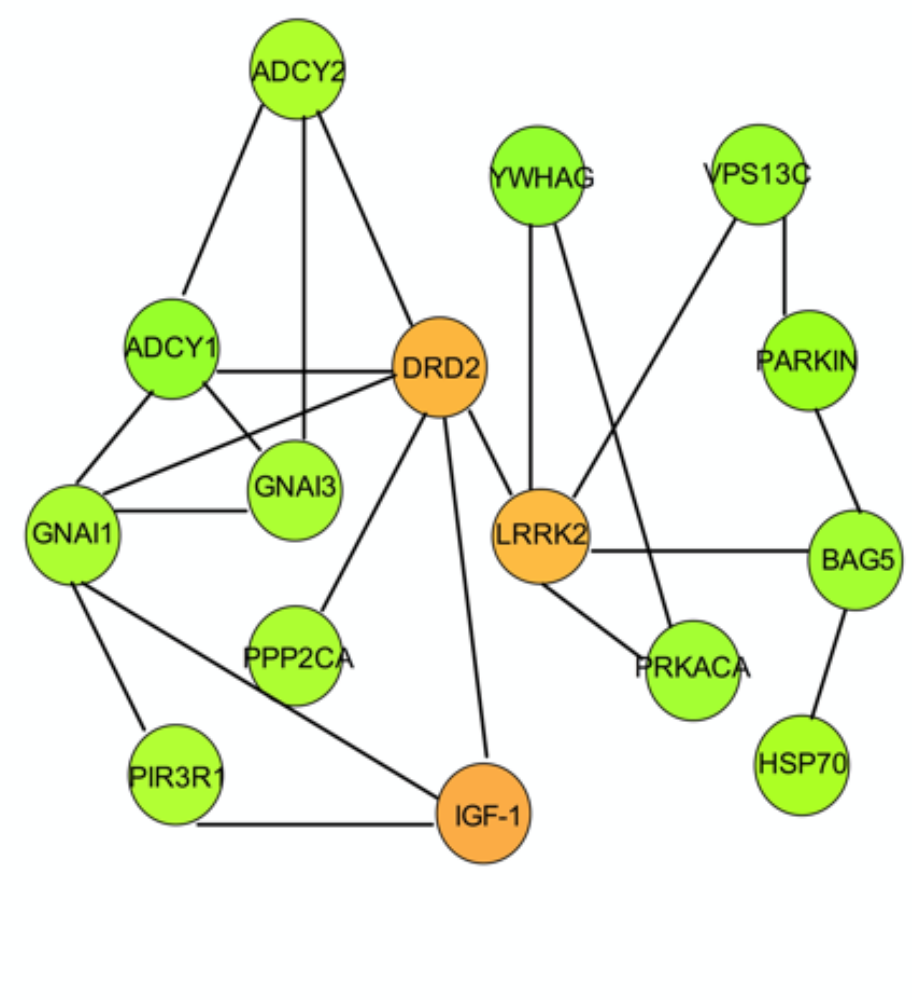

Figure 7

The network of differential expression in gene protein interaction.

\section{Supplementary Files}

This is a list of supplementary files associated with this preprint. Click to download.

- Additionalfile2.doc

- Additionalfile1.doc 